\title{
Ursula Raue, Rechtsanwältin
}

\author{
Juristinnen machen Karriere - wir stellen sie vor
}

Das Interview führte Birgit Kersten, Vorstand des Regionalgruppenbeirats und Vorsitzende der RG Oldenburg im djb, Rechtsanwältin und vereidigte Buchprüferin, Brake, im November 2009 in Berlin. Aufgrund der Aktualität der Themen Missbrauch von Kindern und Kinderpornografie wird dieses Interview in einem der nächsten Hefte durch einen weiteren Beitrag von Ursula Raue, die u.a. vom Jesuitenorden als Beauftragte für Missbrauchsfälle engagiert wurde, ergänzt.

Frau Raue, Sie waren von 1993 bis 1997 1. Vorsitzende des djb, ab 1989 im Bundesvorstand und jahrelang Vorsitzende des Landesverbands Berlin. Welche Themen haben Ihnen dort besonders am Herzen gelegen?

Chronologisch war es so, dass ich zunächst in Berlin mit dem Landesvorsitz begonnen habe. Wir haben 1983 aus Anlass der Arbeitstagung des Juristinnenbundes, die in Berlin stattfand, diese Gruppe gegründet. Die Gruppe hatte einmal existiert, war aber irgendwie nicht mehr präsent. Unsere „Vorkämpferin" Adelheid Koritz-Dohrmann, die damals schon im Bundesvorstand war, hatte ein paar ihrer Kolleginnen und Freundinnen aktiviert und gesagt, wir müssten einen eigenen Landesverband haben. So habe ich den Juristinnenbund kennengelernt. Mich hat dann das, was die Kolleginnen dort machten und was da überhaupt an Arbeit geleistet wurde, sehr beeindruckt.

Ich wurde relativ schnell Vorsitzende dieses Landesverbandes und wurde dann 1989 in den Bundesvorstand gewählt. Es ging also kontinuierlich weiter und das hatte sicher damals auch damit zu tun, dass die Wiedervereinigung und der Umgang insbesondere mit den Juristinnen der DDR für uns in Berlin eine herausragende Rolle spielte. Wir im Westteil von Berlin hatten sehr bald Kontakte mit den Kolleginnen aus dem Ostteil der Stadt. In Bonn, wo der djb damals ansässig war, waren Berlin und die DDR und die Vereinigungsproblematik nicht nur räumlich, sondern auch emotional enorm weit weg. Ich erinnere mich an Situationen, da sah ich manchmal die blanke Angst in den Augen der Kolleginnen, dass wir vom Kommunismus überrollt würden. Nun gehöre ich ja eher nicht in das ganz linke Spektrum. Das hat die Sache vielleicht ein bisschen abgemildert. Aber es war damals eben doch viel Arbeit und ein langer Weg zu gehen.

Ich habe es 1993 für den Bundesvorstand übernommen, den ersten Kongress in einem der neuen Bundesländer zu organisieren. Das war eine sehr gute und intensive Erfahrung und ich habe in der Zeit viel gelernt über die enormen Veränderungen, mit denen die Menschen damals im Umland von Berlin, der ehemaligen DDR und speziell in Thüringen zurechtkommen mussten.

Weimar hat eine ganz eigene Geschichte: Weimar ist Kulturstadt und Weimar steht für die Weimarer Verfassung. Mit viel Mühe und großem persönlichem Einsatz ist es gelungen, das Nationaltheater für die Eröffnungsveranstaltung zu bekommen. Das war ein großes Erlebnis. Die Anstrengung hat sich gelohnt.
Ursula Raue wurde am 4. Juli 1943 in Gütersloh geboren. Nach einer Lehre als Industriekauffrau machte sie Abitur auf dem zweiten Bildungsweg in Bielefeld, studierte anschließend Jura an der Freien Universität Berlin und absolvierte dort auch die beiden Staatsexamen. 1972 verbrachte sie ihre Referendar-Wahlstation in Brüssel bei der EG-Kommission. Einige Jahre war sie als Referentin für Kultur, Schulen, Wissenschaft im Abgeordnetenhaus von Berlin tätig. Seit 1977 ist sie Rechtsanwältin in Berlin. 1976 wurde ihre Tochter, 1978 ihr Sohn geboren. Sie ist ausgebildete Mediatorin. Von 1999 bis 2007 war sie Geschäftsführerin des Landesverbands Berlin im Deutschen Bühnenverein.

1983 gründete sie die Untergruppe Berlin im Deutschen Juristinnenbund. Von 1989 bis 1997 war sie Mitglied im Bundesvorstand des djb, von 1993 bis 1997 als dessen 1. Vorsitzende.

Von 2003 bis 2009 war sie Präsidentin von Innocence in Danger e.V. Deutschland, seit 2008 ist sie Präsidentin des Vereins Sonnenuhr e.V.

Sie ist Mitglied des Kuratoriums der Freien Universität Berlin. Seit 2005 ist sie Mitglied im Vorstand des Vereins der Freunde der Hebräischen Universität Jerusalem. Außerdem ist sie Mitglied von EAF Europäische Akademie für Frauen in Politik und Wirtschaft, Mitglied in verschiedenen Freundesvereinen wie Freunde der Nationalgalerie, des Hebbel-Theaters, des Maxim-Gorki-Theaters.

Sie waren bis vor Kurzem Präsidentin von Innocence in Danger e.V. Wie kamen Sie dazu und was können Sie uns über Ihre Tätigkeit dort berichten?

Dieses Thema wurde im weiteren Freundeskreis an mich herangetragen. Wie vielen Menschen geht es auch mir so, dass sexueller Missbrauch von Kindern mich sehr stark berührt. Ich hatte damals nicht unbedingt vor, wieder ein arbeitsintensives Ehrenamt zu übernehmen. Aber es war tatsächlich so, dass unter denjenigen, die Gründungsmitglieder und sonst in dem Verein waren, meine Profession fehlte. Ich passte da also sehr gut hinein. Außerdem war auch die Tatsache damals sehr hilfreich, dass ich zuvor Präsidentin des djb war. Das hat uns in den Justizministerien die Türen geöffnet und wir sind auf Verständnis gestoßen. Den anderen, die damals mit im Vorstand waren, war das zu dem Zeitpunkt so nicht möglich. Diese Kombination der verschiedenen Berufe und Tätigkeitsfelder war interessant und die Zusammenarbeit in dieser Gruppe war sehr effizient.

Innocence in Danger wurde 1999 in Paris gegründet, um Kinder gegen Missbrauch und sexuelle Ausbeutung zu schützen. 
Die Organisation ist heute in 28 Ländern aktiv. Welche Themen standen in den ersten Jahren im Vordergrund? Wie wurde dort gearbeitet?

Tatsächlich war es so, dass 1999, als Innocence in Danger in Frankreich gegründet wurde, schon das Thema Internet im Raum stand. Es gab damals eine Unesco-Konferenz, die sich mit diesem Thema beschäftigte. Am Ende hieß es, dass man sich in zehn Jahren wieder treffen müsse. Eine der Teilnehmerinnen, eine Schweizerin, sagte dann, zehn Jahre können wir nicht warten. Sie war die Initiatorin dieser ganzen Organisation und hat Innocence in Danger in Paris gegründet. Es gab dann eine Stiftung in der Schweiz, gewissermaßen als Dachorganisation, und von dort aus sind die Einrichtungen in den einzelnen Ländern entstanden.

Wie geht der Verband vor? Mit wem arbeitet die Organisation zusammen? Gehören auch Psychologen dazu und welche anderen Disziplinen sind vertreten?
Sache brachte. Auch die Antwort auf die Frage: „Wie kann man dem begegnen? “ wurde klar: „Nur, indem alle Beteiligten zusammenarbeiten."

Es ist natürlich nach wie vor ein schwieriges Thema, weil es bei den Vertretern der Provider nicht immer das nötige Engagement gibt. Denn damit wird einfach sehr viel Geld verdient.

Man muss sich auch darüber im Klaren sein, dass Pornografie, wenn es sich um Erwachsene handelt, nicht verboten ist. Wenn allerdings Kinder involviert sind, ist es anders. Innocence in Danger hat immer darauf Wert gelegt, auch klarzustellen, dass all das, was im Internet steht, was auf Fotos zu sehen ist und für horrende Geldsummen verbreitet wird, immer einen konkreten Missbrauch als Hintergrund hat. Jedes Kind, das auf einem Film oder auf einem Foto zu sehen ist, ist auf bestialische und fürchterliche Weise missbraucht worden. Und da muss man natürlich auch ansetzen, das heißt, es geht vor allen Dingen darum, aufzurütteln und zu sagen, passt besser auf eure Kinder auf, macht sie stark, seid euch der Tatsache

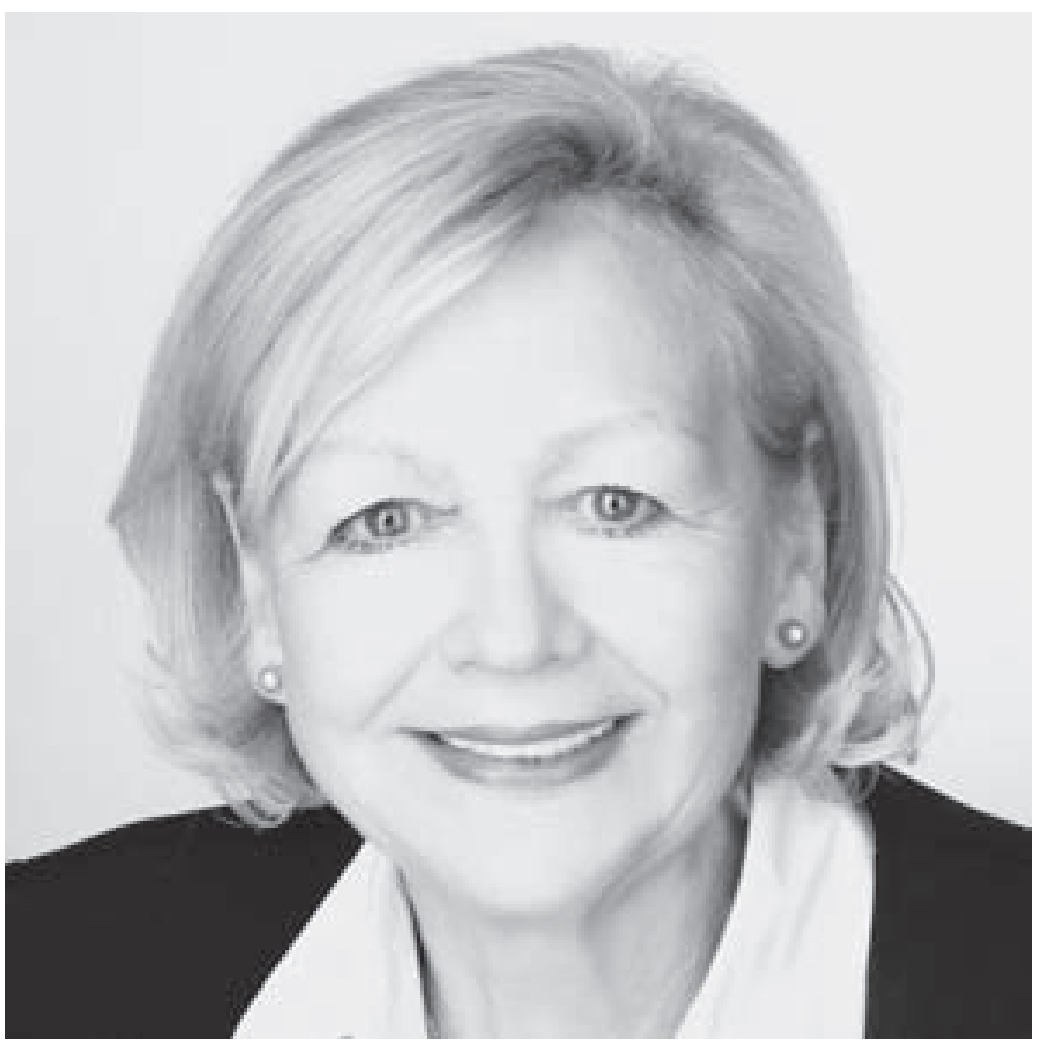

Die Internetkriminalität nimmt einen großen Raum ein. Die deutsche Sektion von Innocence in Danger wurde im Dezember 2002 gegründet, 2003 gab es eine erste interdisziplinäre Konferenz, die damals hier im Berliner Abgeordnetenhaus stattfand. Da haben wir versucht, alle Disziplinen, alle Beteiligten an diesem Verfahren dabei zu haben und das ist auch relativ gut gelungen. Es gab Vertreter von Banken - weil immer auch Kreditkarten eine Rolle spielen - und Providern und es gab natürlich auch Psychologen und Psychologinnen, die einschlägige Erfahrungen hatten. Dieser Austausch war damals tatsächlich sehr fruchtbar, weil er Bewegung in die bewusst, dass es diese schrecklichen Dinge gibt.

Es gibt eine hoch qualifizierte Frau, eine Psychologin und Psychotherapeutin, die 15 Jahre lang mit schwerst traumatisierten Kindern gearbeitet hat. Durch die Unterstützung von Aktion Mensch - und möglicherweise auch demnächst des Familienministeriums - haben wir immer wieder Geld akquirieren können, mit dem wir als Verein in der Lage sind, diese Frau seit gut zwei Jahren als Geschäftsführerin zu beschäftigen und sie auch angemessen zu bezahlen. Das sind natürlich immer Verträge, die sich über einen bestimmten Zeitraum und über Projekte entwickeln, aber wir haben die Hoffnung, dass 
es weitere Projekte gibt und sind einfach sehr froh, dass wir diese großartige Fachfrau haben.

\section{Geht es bei der Arbeit von Innocence in Danger ausschließ- lich um Aufklärung oder gibt es auch Projekte für traumati- sierte Kinder?}

Es ist mir wichtig, darauf aufmerksam zu machen, dass wir inzwischen von Erhebungen wissen, dass ein Kind etwa acht Mal in irgendeiner Form „Hilf mir“ sagt, also einen Hilferuf aussendet, wenn es bedrängt wird, wenn es sexuellem Missbrauch ausgesetzt ist. Das Problem ist, dass die Umgebung es nicht versteht. Da sieht es Innocence in Danger als eine wichtige Aufgabe an, dass diese Zeichen besser verstanden werden und dass die Sensibilität für die Nöte der Kinder, die in so einer Missbrauchsgeschichte verfangen sind, erhöht wird.

Neben der Aufklärung geht es tatsächlich auch um Projekte für traumatisierte Kinder. An allererster Stelle ist da unsere Kunstwoche, die 2009 zum drittenmal stattgefunden hat. Es sind immer maximal acht bis zehn Kinder dabei, die zusammen mit einem Elternteil oder einer Betreuerin aus dem Heim, in dem sie leben, eine Woche lang so richtig entspannen können. Dabei sind Künstler, mit denen die Kinder fotografieren. Es wird gemalt und musiziert, die Kinder werden verpflegt und es ist einfach so eine ganz lockere Atmosphäre, in der die Kinder erstaunlich fröhlich werden und aufblühen. Die Kinder werden auch von erfahrenen Therapeutinnen begleitet. Wenn ich dabei bin, stelle ich eigentlich immer mit Erstaunen fest, wie die Kinder sich da in so kurzer Zeit eine neue Welt erschließen. Man kann sagen, sie lernen dort etwas, was sie nie wieder verlernen, es bleibt wirklich hängen. Das ist unglaublich zu sehen und es stimmt auch überein mit den Erfahrungen der Betreuerinnen, also der Mütter oder Erzieherinnen, die dabei sind. Sie sagen, dass die Kinder sich wirklich verändern.

Die erste Kunstwoche haben wir mit der Spende einer Stiftung aus Süddeutschland über 10.000 Euro finanzieren können. Diese Stiftung hatte uns das Geld für eine ähnliche Veranstaltung in der Schweiz zur Verfügung gestellt, die dann aber nicht stattfand. Ich habe die Stiftung gefragt, ob wir das Geld für eine ähnliche Sache in Brandenburg ausgeben dürfen. Sie waren einverstanden. Eines unserer Vorstandsmitglieder hat uns sein Landhaus in Brandenburg zur Verfügung gestellt und mit diesen 10.000 Euro haben wir die wesentlichen Ausgaben finanzieren können. In dieser Kunstwoche sind Arbeiten auf Papier entstanden, Malerei und Fotoarbeiten. Diese Arbeiten wurden in einer Auktion versteigert. Dabei wurden dann knapp 20.000 Euro erzielt und damit haben wir die nächste Woche finanziert. Und so ist es bisher immer gewesen. Die Kunstwoche hat dreimal stattgefunden. Die zweite und dritte Kunstwoche wurde jeweils aus Erlösen der Bilder der ersten und zweiten Auktion finanziert. Die Kunstwochen tragen sich vollständig selbst.

Ein Vortrag von Innocence in Danger 2008 für Eltern und Fachleute hatte das Thema „blogger, twitter, jabber und co ...“.
Ich habe versucht, mit Hilfe von Wikipedia zu verstehen, wie Jabber funktioniert. Wie geht es Ihnen damit? Inwieweit können Kinder und Jugendliche dadurch gefährdet werden?

Ich stehe auch immer wieder hilflos vor diesen Ausdrücken, die sich da neu etablieren und muss mich erkundigen, um was es sich handelt. Innocence in Danger hat seit 2008 ein Projekt, das wiederum durch Aktion Mensch finanziert wird. Es geht dabei um Internet, Handy, iPod \& Co. und um die Gefahren, die von diesen Techniken und diesen neuen Möglichkeiten für Schüler, Kinder und Jugendliche ausgehen. Das Ziel des Projektes ist es, Eltern, Lehrern, Lehrerinnen und auch den Kindern Regeln an die Hand zu geben für den Umgang mit den neuen Geräten und Techniken. Es heißt „Smart User" und wird gerade entwickelt. Das Projekt läuft bis Ende 2010. Ende 2010 werden wir also Ergebnisse vorstellen können. Gearbeitet wird an dem Projekt mit Schülern und Schülerinnen von drei verschiedenen Schulen in Köln, darunter auch eine Schule für Gehörlose, weil auch diese Schülerinnen und Schüler natürlich mit Internet und Handy arbeiten können.

In den Vorgesprächen mit den Schülerinnen und Schülern hat sich herausgestellt, dass 80 Prozent von ihnen schon sexuelle Anmache im Internet erlebt haben und dass die Kinder nicht Bescheid wissen über das, was sie mit ihren Handys tun. Es ist zur Zeit zum Beispiel absolut üblich, alles Mögliche auf Klassenreisen mit dem Handy zu filmen. Dazu gehört natürlich auch das, was wir früher Doktorspiele nannten, d.h. sich mit dem Körper und der Sexualität des anderen oder des eigenen Geschlechts auseinanderzusetzen. Sicher ist die Sexualisierung in der täglichen Umgebung heute intensiver als früher; hinzu kommt, dass die Fotos und Videos dank der technischen Ausstattung der Schülerinnen und Schüler dann häufig sofort in der Klasse, in der Gruppe herumgeschickt werden und dann steht das alles im Internet und ist für alle Zeiten dort. Man bekommt es nie wieder heraus. Das ist die Gefahr. Es ist ein ganz wesentlicher Teil der Arbeit von Innocence in Danger, hier zu versuchen, Regeln zu etablieren und den Kindern klarzumachen, in welche Gefahr sie sich begeben, wenn sie sich die Konsequenzen nicht bewusst machen.

\section{Der Deutsche Bundestag hat im Juni 2009 das Gesetz zur Zu- gangserschwerung zu Kinderpornografie im Internet be- schlossen. Halten Sie das Gesetz für ausreichend?}

Diese Zugangserschwerung zu Kinderpornografie im Internet ist ein wichtiger erster Schritt. Fachleute sind ja entsetzt und sagen, dieses Stoppschild, das hilft uns überhaupt nicht weiter, das kann sofort umgangen werden und die Leute umgehen das auch. Ich glaube aber, man muss hier zwischen dem informierten und dem weniger informierten Benutzer unterscheiden. Der ganz normale Mensch, der an so eine Seite kommt, wird sich wahrscheinlich erst einmal abschrecken lassen. Dass wir in dieser Frage die Provider mit auf unsere Seite bekommen müssen, damit sie von sich aus tätig werden, ist das Ziel, das wir erreichen müssen. Aber so weit sind wir leider noch nicht. 
Sind die ermittelnden Behörden in Deutschland hinreichend ausgestattet, um effektiv und schnell genug zu ermitteln? Ich nehme mal an, dass es keine Behörde gibt, die sagt, wir sind zu gut oder hinreichend ausgestattet. Das trifft natürlich auch auf die ermittelnden Behörden hier zu. Ganz klar. Nach meiner Beobachtung und nach dem, was wir wissen, ist es tatsächlich so, dass sie nicht hinreichend ausgestattet sind. Es müssen Millionen von Bildern kontrolliert werden und es ist auch eine ganz schreckliche Arbeit, wenn man sich diese Fotos und Filme dauernd ansehen muss. Eigentlich brauchen die Leute, die das tun, auch eine psychologische Betreuung. Stattdessen wissen wir, dass sie in den Polizeibereichen eher eine Abwertung erfahren. Die Kollegen sagen dann, ihr schaut den ganzen Tag Schmuddelkram an und wir müssen die Arbeit draußen machen. Also die Anerkennung dieser Arbeit und das Bewusstsein für deren Wichtigkeit fehlt häufig noch.

Nach $\S 6$ StGB gilt das Weltrechtsprinzip, d.h. das nationale Strafrecht ist auch auf Sachverhalte anzuwenden, die keinen spezifischen Bezug zum Inhalt haben, bei denen also weder der Tatort im Inland liegt noch der Täter oder das Opfer die Staatsangehörigkeit des betroffenen Staates besitzen. Die Verbreitung kinderpornografischer Schriften gehört hierzu. Kooperiert das Ausland? Ist es ohne Weiteres möglich, an ausländische Server heranzukommen?

Die Staaten, in denen viele dieser Taten passieren, sind nur in Grenzen kooperativ und da ist natürlich noch sehr viel zu tun. Gerade in der internationalen Absprache fehlt es an der Bereitschaft und natürlich auch an den Möglichkeiten, auf Behörden oder Einzelpersonen den nötigen Druck auszuüben.

Für die Verbreitung, den Erwerb und den Besitz von kinderpornografischen Schriften beträgt die Strafe maximal fünf Jahre ( $\$ 184$ b StGB). Somit liegt der Strafrahmen bei dem des Diebstahls oder der Urkundenfälschung. Sind die Strafen hoch genug?

Nein, die Strafen für den Erwerb, den Besitz und die Verbreitung von kinderpornografischen Schriften sind nicht hoch genug, weil hinter jeder Dokumentation, hinter jeder Darstellung, die als Bild verbreitet wird, immer der Missbrauch eines jungen Menschen, eines Kindes steckt. Dieser Strafrahmen trägt der Schwere der Taten, die zur Herstellung dieser Bilder begangen wurden, überhaupt nicht Rechnung.

Neben dem Internet findet Pornografie auch Verbreitung durch Handys und iPods. Wie können Kinder und Jugendliche dort geschützt werden?

Genau dies wird mit dem „Smart User“-Projekt versucht. Da werden die Regeln erarbeitet und wir hoffen alle, dass wir spätestens in einem Jahr etwas vorstellen können, mit dem man wirklich weiterkommt.

Wie wird das Projekt N.I.N.A. angenommen, die erste bundesweite Telefonnummer für Erwachsene, die Informationen und Hilfe zum Thema sexueller Missbrauch benötigen?
Vor zwei Jahren gab es hier in Berlin eine Kampagne, die ein großes Werbeunternehmen dadurch unterstützt hat, dass überall Plakate aufgehängt wurden. Die Folge war, dass N.I.N.A. gerade aus Berlin und Brandenburg mit Anfragen überlaufen wurde. Ähnlich war es mit einer Postkartenaktion, die das Projekt bekannt gemacht hat.

Zur Zeit bemühen sich unsere Geschäftsführerin und der Vorstand darum, eine solide Finanzierung für N.I.N.A. zu finden. N.I.N.A. ist ein Angebot für Erwachsene, die den Verdacht haben, es könne in ihrem Umfeld sexueller Missbrauch passieren. So wird es auch angenommen.

Inzwischen läuft sehr viel über E-Mails, denn das telefonische Angebot steht immer noch nicht die ganze Woche über zur Verfügung, weil wir bisher nicht genügend Mittel haben, zusätzliche Mitarbeiterinnen für die Arbeit am Telefon auszubilden. Für eine qualifizierte Beratung ist nicht nur eine psychotherapeutische Ausbildung, Erfahrung im Umgang mit Opfern von sexueller Ausbeutung, sondern auch eine spezielle Ausbildung für die Arbeit am Telefon und begleitende Supervision erforderlich. Das ist relativ personalkostenintensiv und wir hoffen, ein Unternehmen oder eine Stiftung zu finden, mit dessen oder deren Hilfe N.I.N.A. weiter finanziert werden kann und es eines Tages als Pilotprojekt vom Familienministerium übernommen und dann auch finanziert wird.

\section{Welchen Schwerpunkt hat Ihre Tätigkeit als Rechtsanwältin?}

Ich habe mich nach einer Familienpause relativ bald mit Mediation beschäftigt. Die Mediation war damals neu, sie wurde vor allen Dingen in Deutschland von zwei Amerikanern angeboten, die ihre Seminare und Kurse in Heidelberg und Berlin gehalten haben. Ich fand das sehr bereichernd und so habe diese Ausbildung abgeschlossen und bin als Mediatorin auch tätig. Von 1999 bis 2008 war ich Geschäftsführerin des Landesverbands Berlin im Deutschen Bühnenverein. Das war keine ehrenamtliche Tätigkeit. In der Zeit habe ich mich vorrangig mit Theater und mit Fragen des Theaterrechtes beschäftigt. Die Mediation war auch in diesem Bereich sehr hilfreich.

Der djb ist auch ein Verband für Volks- und Betriebswirtinnen. Können Sie sich an eine Zusammenarbeit im Vorstand oder in einer Kommission erinnern, in der die Kenntnisse von Wirtschaftswissenschaftlerinnen besonders hilfreich waren? Die Kenntnisse von Wirtschaftswissenschaftlerinnen wurden im Berliner Landesverband und auch im Bundesverband in der Weise genutzt, dass wir unter diesen Kolleginnen oft sehr zuverlässige Schatzmeisterinnen gefunden haben. Und dann gab es natürlich auch den Familienlastenausgleich und die Steuerrechtskommission. Alles, was sich mit wirtschaftlichen Fragen befasste, fiel auch in die Kompetenz der Wirtschaftswissenschaftlerinnen. Da haben sie auch viel zur Arbeit beigetragen. Im Übrigen lag die Kernkompetenz des djb immer im Familienrecht und dann auch im Strafrecht. Das sind Themenkreise, die Wirtschaftswissenschaftlerinnen in der Regel nicht berühren, das heißt, sie haben damit im professionellen Bereich normalerweise nichts oder nur wenig zu tun. 
Ein ganz neues Gebiet für ein Engagement von Wirtschaftswissenschaftlerinnen ist die Beteiligung von Frauen in den Aufsichtsräten. Da sind unsere Kolleginnen von den wirtschaftswissenschaftlichen Fakultäten natürlich sehr gefragt und da brauchen wir ihre Kenntnisse und ihr Wissen.

\section{Auch zum Thema Entgeltgleichheit.}

Ja, bei allem, was mit den Karrieren und der Situation von Frauen innerhalb der Wirtschaft zu tun hat, sind uns die Wirtschaftswissenschaftlerinnen eine riesige Hilfe.

Sie engagieren sich sehr vielfältig ehrenamtlich, neben Innocence in Danger e.V. beispielsweise im Freundeskreis Hebbeltheater e.V. Sie sind Mitglied im Vorstand der Freunde der Hebräischen Universität Jerusalem und Mitglied des Kuratoriums der FU Berlin. Sie interessieren sich neben dem Theater für Musik, bildende Kunst und Literatur. Erzählen Sie uns zum Abschluss etwas über Ihre Unterstützung von Sonnenuhr e.V.

Im Laufe meines Lebens haben sich verschiedene Engagements angesammelt. Aber seit einiger Zeit liegt mir ganz besonders wieder das Projekt Sonnenuhr am Herzen. Ich habe diese Truppe 1990 kennengelernt, als sie sich gründete. Es gibt sie nunmehr seit fast 20 Jahren. Das sind zwei Theatergruppen, in denen Menschen mit geistiger Behinderung - viele davon mit Downsyndrom - unter der Anleitung von professionellen Theaterleuten Stücke der klassischen Theaterliteratur oder auch eigene Produktionen spielen. Ich erinnere mich genau, was damals auf die Bühne kam und was für Stücke da gespielt wurden. Es war eindrucksvoll, mit welcher Intensität und Hingabe diese Menschen aller Altersklassen sich als Schauspieler oder bildende Künstler ausdrücken und mitteilen konnten.
Und wenn ich mir anschaue, was sie heute können, dann ist es unglaublich faszinierend zu sehen, welch ein Potential in diesen Menschen steckt, denen die Gesellschaft ja eigentlich noch nicht einmal eine Ausbildung anbieten kann, sondern die in der Regel in Behindertenwerkstätten unterkommen, dort Tüten kleben oder irgendwelche Körbe flechten. Heute sieht man, was dort mit unendlich viel Geduld, Einsatz und Liebe vollbracht oder geschehen ist, dass einige von ihnen jetzt wunderbar singen und dass da schauspielerische Potentiale vorhanden sind, von denen vor 20 Jahren niemand etwas geahnt hat. Ich finde, dass diese Erfahrungen und Erkenntnisse einfach nicht verloren gehen dürfen.

Es gibt da eine Schauspielerin, eine junge Frau Ende 20 mit Downsyndrom, von der mir die Mutter vor Jahren erzählt hat, was ihr die Ärzte gesagt hatten: Sie werde mit diesem Kind nie kommunizieren können und es werde daliegen und wahrscheinlich sowieso bald sterben. Heute singt dieses Mädchen eine so wunderbare Seeräuber-Jenny, dass es mir immer kalt den Rücken herunterläuft. Das ist - nach Lotte Lenya - das Schönste und Beste, was ich je dazu gehört habe. Oder eine der Schauspielerinnen ist inzwischen in der Fernsehserie Tatort in einer Hauptrolle aufgetreten. Für mich ist es eine wichtige Aufgabe, dieses Projekt in die nächste und in die übernächste Generation zu bringen.

Das Theater dieser Truppe, die auch Foto- und Kostümwerkstätten hat und dort alles selbst herstellt, hat den wunderbaren Namen „RambaZamba“ und es ist in der Fachwelt - auch international - inzwischen sehr bekannt und anerkannt.

Frau Raue, ich danke Ihnen für das Gespräch.

\section{Impressum}

\author{
Schriftleitung: \\ Anke Gimbal, Rechtsassessorin (V.i.S.d.P.) \\ Juliane Lindner \\ Redaktionsanschrift: \\ Deutscher Juristinnenbund e. V. \\ Anklamer Str. 38 \\ 10115 Berlin \\ Telefon: 030 443270-0 \\ Telefax: 030 443270-22 \\ E-Mail: geschaeftsstelle@djb.de
}

\section{Druck und Verlag:}

Nomos Verlagsgesellschaft mbH \& Co. KG

Waldseestr. 3-5

D-76530 Baden-Baden

Telefon: 07221 2104-O

Telefax: 07221 2104-27

\author{
Anzeigenverwaltung und \\ Anzeigenannahme: \\ Sales friendly, Verlagsdienstleistungen \\ Bettina Roos \\ Siegburgerstr. 123 \\ D-53229 Bonn \\ Telefon: 0228 97898-o \\ Telefax: 0228 97898-20 \\ E-Mail: roos@sales-friendly.de
}

Die Zeitschrift sowie alle in ihr enthaltenen einzelnen Beiträge und Abbildungen sind urheberrechtlich geschützt. Jede Verwertung, die nicht ausdrücklich vom Urheberrechtsgesetz zugelassen ist, bedarf der vorherigen Zustimmung des Verlages.

Namentlich gekennzeichnete Artikel müssen nicht die Meinung des Herausgebers oder der Schriftleitung wiedergeben. Unverlangt eingesendete Manuskripte - für die keine Haftung übernommen wird - gelten als Veröffentli- chungsvorschlag zu den Bedingungen des Verlags. Es werden nur unveröffentlichte Originalarbeiten angenommen. Die Verfasser erklären sich mit einer nicht sinnentstellenden redaktionellen Bearbeitung einverstanden.

\author{
Erscheinungsweise: \\ vierteljährlich \\ Bezugspreis 2010: \\ jährlich 48,-€, Einzelheft 13,-€
}

Alle Preise zzgl. Vertriebs-Direktbeordnungsgebühren inkl. MWSt.;

Bestellungen nehmen entgegen:

Der Buchhandel und der Verlag; Kündigung jeweils drei Monate zum Kalenderjahresende. Zahlungen jeweils im Voraus an:

Nomos Verlagsgesellschaft, Postbank Karlsruhe, Konto 7363651 (BLZ 66010075) oder Stadtsparkasse Baden-Baden, Konto 5002266 (BLZ 66250030). 